\title{
Tackling the burden of injury in Australasia: developing a binational trauma registry
}

Tamzyn M Davey, CliffW Pollard, Leanne M Aitken, Mark Fitzgerald, Nicholas Bellamy, Daniel Cass, Peter D Danne, William M Griggs, Peter A Cameron, Robert N Atkinson, James Hamill, Sudhakar Rao, Drew B Richardson and Christine O'Connor

T rauma registries are an essential means of measuring the extent of injury through the collation and effective use of detailed data on injured patients. They also inform injury prevention strategies, monitor changing patterns of injury and associated management, and facilitate comparison of management across institutions. ${ }^{1,2}$ Trauma registries also provide an important source of data for determining the financial costs of injury. ${ }^{3,4}$ The data routinely collected by trauma registries on all aspects of injured patients, from before hospitalisation to patient outcome at hospital discharge, provide the opportunity for improving the management of injured patients and help in the planning of trauma services..$^{1-3}$ The capacity to audit and improve injury management is a fundamental component of any trauma system, and it is arguably the main impetus for continued funding of the current Australasian trauma registries.

\section{Trauma registries in Australia and New Zealand}

Trauma registries have existed in Australia and New Zealand in either a state-based or individual hospital-based form for more than a decade. Currently in Australia, there are state-based trauma registries in New South Wales, Queensland, South Australia and Victoria. Western Australia has hospital-based trauma registries at Fremantle, Princess Margaret, Royal Perth, and Sir Charles Gairdner hospitals. Presently, there are no trauma registries in either of the territories. In New Zealand, registries operate from Auckland City and Middlemore hospitals.

Performance improvement at the current trauma registry level is not systematic, but takes place in most existing trauma registries across Australasia. It most commonly takes the form of a system of alerts of instances of injury management that did not meet the consensus-derived "optimal" level of care. Identified cases are then reviewed by clinicians at each hospital and, where the reasons for "suboptimal" care were preventable, improvements are made to prevent or reduce the likelihood of such an event recurring. Improvements may be made through, for example, educating clinical staff, changing clinical processes and practices, and lobbying for increased funding for equipment or services.

Despite ongoing (albeit ad-hoc) auditing of the performance of injury management in trauma systems across Australasia, there is currently no way of adequately benchmarking the standard of injury management across the Tasman, between states, and, in some cases, within states. Other than benchmarks for probability of death, which are based on estimations developed in the United States 20 years ago, there are no normative data to allow routine benchmarking of the performance of injury management in Australasian trauma systems.

\section{Towards a binational trauma registry}

In 2003, the vision for a binational pool of data on injury of a defined severity took shape in the form of the Australian and New Zealand National Trauma Registry Consortium (NTRC). The NTRC

\section{ABSTRACT}

- Existing trauma registries in Australia and New Zealand play an important role in monitoring the management of injured patients.

- Over the past decade, such monitoring has been translated into changes in clinical processes and practices.

- Monitoring and changes have been ad hoc, as there are currently no Australasian benchmarks for "optimal" injury management.

- A binational trauma registry is urgently needed to benchmark injury management to improve outcomes for injured patients.

MJA 2006; 185: 512-514

was a collaborative initiative of the Royal Australasian College of Surgeons, the Centre of National Research on Disability and Rehabilitation Medicine, and the Australasian Trauma Society. The NTRC "project" was initially funded jointly by the above institutions, and since 2005, the NSW Institute of Trauma and Injury Management has also provided funding. An executive and steering committee with representatives from all the relevant states and/or hospitals was established to facilitate the decision-making process and advance the NTRC towards the development of a binational trauma registry. The NTRC has fulfilled its primary goal of linking together all the existing trauma registries in Australia and New Zealand, as well as other key players who have an interest in improving the management of injury in Australasia. The commitment to the NTRC project is evident in the ongoing willingness of all current registries in Australasia to submit data, which has, to this point, enabled the production of two annual reports.

Both annual reports were based on deidentified aggregate data describing aspects of injury, including demographic characteristics, injury cause and description, and patient outcome (see Box). Variables that were included were those that had the least variability with regard to definition and were common to most participating registries. Only patients who were admitted to a participating trauma registry hospital with an Injury Severity Score (ISS) greater than 15 were included. The ISS is calculated using the Abbreviated Injury Scale scoring system, which was first introduced in 1969 by the American Association for the Advancement of Automotive Medicine, and is the most widely used anatomical injury severity scale in the world. ${ }^{6}$ The ISS gives an indication of the severity of the combination of injuries sustained by a patient, and is calculated as the sum of the square of the highest Abbreviated Injury Scale code in each of the three most severely injured body regions. ${ }^{6}$ The NTRC's decision to include only injury cases with an ISS of greater than 15 was made to enable inclusion of all the existing trauma registries (some of which only collect cases with an ISS greater than 15), and because this is consistent with international trends for inclusion in trauma registries. Both annual reports 
are available on the NTRC website (http://www.uq.edu.au/ntrc/) under the heading "Publications".

\section{The urgent need for a binational trauma registry}

The number of injuries over the 2 years of reported data provides strong evidence of a high burden of injury in Australasia, particularly as the estimate excludes patients admitted with an ISS of 15 or less, and patients who were treated by a general practitioner, but not subsequently admitted to hospital. The limitations of the descriptive aggregate dataset include not having access to primarylevel data to enable more meaningful analysis, heterogeneity in rules governing data inclusion at different trauma registries, and varying definitions for "common" variables. It is difficult to share individual-level data under current ethical regulations, because approval from every participating hospital is required. A binational trauma registry would be in a stronger position than the current NTRC project to negotiate access to deidentified primary-level data under quality assurance regulations. This would facilitate the monitoring of injury management and provide a more meaningful dataset for research purposes.

The development of a binational trauma registry in Australasia, as a repository of pooled data from the current trauma registries to allow widespread benchmarking of injury management, is thus timely. In addition, it would enable us to describe the incidence, management or outcome of injured patients in the Australasian region - which is not currently possible. Accurate and complete description of injured patients would allow more effective planning and provision of services, and would inform prevention strategies.

The primary purpose of a binational trauma registry would be to provide the existing registries and their associated trauma systems with benchmarks of injury management against which they could assess their own performance and implement strategies to improve care to meet these benchmarks. A binational trauma registry would also be essential for quantifying the extent of injury in Australasia, providing a means of monitoring the changing patterns of injury and determining the financial costs. Such a registry would also make available a large, comprehensive dataset on injury for research and comparison with international injury datasets. Also of invaluable benefit would be the relationships which will develop in the common pursuit of improving injury management, and which will ideally result in a unified and powerful lobby to governments for improved legislation with regard to injury.

\section{The future}

Until long-term funding for the development and maintenance of a binational trauma registry is secured, the NTRC will continue to engage the commitment of existing registries in Australasia by collecting aggregate data for annual reports. The NTRC will also continue to work toward securing formal approval from the relevant legislative bodies and support from all the registries for access to primary-level data. A working party with representatives from all the registries has also been established to address the logistical issues related to including, defining and collecting data to form the basis of the binational trauma registry. Furthermore, the NTRC will encourage researchers from existing entities throughout Australasia to undertake collaborative research focusing on the management of injured patients that informs policy and practice,
Summary of injury characteristics (Injury Severity Score $>15$ ) obtained from Australasian trauma registries in 2003

- 5837 injured patients with an Injury Severity Score greater than 15 were identified.

- The injuries resulted in a mortality rate of $15 \%$ before hospital discharge.

- Males comprised $72 \%$ of the cohort.

- Survival rates to hospital discharge for females and males were about equal ( $82 \%$ and $85 \%$, respectively).

- More people aged between 15 and 24 years (1303) were injured in 2003 than those in any other age group.

- The highest death rate was recorded in those aged over 85 years (41\%).

- Road traffic crashes accounted for $54 \%$ of all major injuries for which a specific external cause of injury was recorded.

- Within the "Road traffic crash" subcategory, pedestrians were most likely to die after admission for the treatment of their injuries (21\% of injured pedestrians did not survive to hospital discharge).

- The average length of hospital stay after admission for the treatment of an injury was 16 days.

- $49 \%$ of patients required admission to an intensive care unit for treatment of their injuries.

- Patterns and distributions of injury in terms of injury characteristics, patient management, and outcomes were largely similar from 2002 to 2003.

and is capable of improving outcomes for injured people in Australasia.

The first study proposal for research collaboration has already been accepted by the NTRC, and aims to estimate the age-specific and injury-specific probability of survival to discharge for patients recorded by the existing registries throughout Australasia. In the absence of a binational primary-level dataset, the researchers will apply for ethical approval to conduct the research and collect data separately from each registry. This study will provide invaluable insight into the data collection processes of the participating registries, as well as the methodological issues related to collecting data from multiple registries.

\section{Conclusion}

Existing trauma registries have functioned effectively throughout Australasia to monitor the performance of injury management in their relevant jurisdictions. However, this process is ad hoc and not systematic. The NTRC (Australia and New Zealand) was established to link all existing trauma registries and to seek commitment toward the development of a binational trauma registry, to allow Australasia-wide benchmarking of injury management. If the vision of improved outcomes for trauma patients in Australasia is to be realised, and the current deficits in performance benchmarking addressed, long-term funding for the development and maintenance of a binational trauma registry is urgently required.

\section{Acknowledgements}

We thank participating trauma registries, which include the central registries: New South Wales Institute of Trauma and Injury Management, Queensland Trauma Registry, South Australian Trauma Registry and the Victorian State Trauma Registry; and the individual hospital registries at Auckland City 
Hospital (New Zealand), Fremantle Hospital (Western Australia), Princess Margaret Hospital (Western Australia), Royal Perth Hospital (Western Australia), Sir Charles Gairdner Hospital (Western Australia) and Waikato Hospital (New Zealand). The National Trauma Registry Consortium project is funded by the Centre of National Research on Disability and Rehabilitation Medicine at the University of Queensland, the Royal Australasian College of Surgeons, the Australasian Trauma Society and the New South Wales Institute of Trauma and Injury Management.

\section{Competing interests}

None identified.

\section{Author details}

Tamzyn M Davey, BSocSci(Hons), Project Officer ${ }^{1}$

Cliff W Pollard, MB BS, FRACS, Chairman, Trauma Systems

Performance Improvement and Registries Sub-Committee ${ }^{2}$

Leanne M Aitken, RN, PhD, Professor of Critical Care Nursing ${ }^{3}$

Mark Fitzgerald, MB BS, FACEM, MRACMA, Director ${ }^{4}$

Nicholas Bellamy, MB ChB, MD, FRCP, Director

Daniel Cass, BSc(Med), FRCS, FRACS, Director ${ }^{5}$

Peter D Danne, MD, FRACS, FACS, Associate Professor ${ }^{6}$

William M Griggs, DipAvMed, FANZCA, FJFICM, Director ${ }^{7}$

Peter A Cameron, MB BS, MD, FACEM, $\mathrm{Head}^{8}$

Robert N Atkinson, DCH, FRACS, FAOrth, Chairman, South Australia

Trauma Committee ${ }^{2}$

James Hamill, MB ChB, FRACS, Paediatric Surgeon?

Sudhakar Rao, MB BS, FRACS, Head ${ }^{10}$

Drew B Richardson, MB BS, FACEM, Director ${ }^{11}$

Christine O'Connor, BPsyc(Hons), PhD, Research Officer ${ }^{12}$

1 Centre of National Research on Disability and Rehabilitation Medicine, University of Queensland, Brisbane, QLD.
2 Royal Australasian College of Surgeons, Brisbane, QLD.

3 Griffith University and Princess Alexandra Hospital, Brisbane, QLD.

4 Emergency and Trauma Centre, The Alfred Hospital, Melbourne, VIC.

5 New South Wales Institute of Trauma and Injury Management, Sydney, NSW.

6 Department of Surgery, University of Melbourne, Melbourne, VIC.

7 Trauma Service, Royal Adelaide Hospital, Adelaide, SA.

8 Victorian State Trauma Registry, Department of Epidemiology and

Preventive Medicine, Monash University, Melbourne, VIC.

9 Starship Children's Hospital, Auckland, New Zealand.

10 Department of General Surgery, Royal Perth Hospital, Perth, WA.

11 Emergency Medicine, Canberra Hospital, Canberra, ACT.

12 Queensland Health, Brisbane, QLD.

Correspondence: t.davey@uq.edu.au

\section{References}

1 McLellan B. A Canadian National Trauma Registry: the time is now. J Trauma 1997; 42: 763-768.

2 Condello AS, Hancock BJ, Hoppensack M, et al. Pediatric trauma registries: the foundation of quality care. J Pediatr Surg 2001; 36: 685-689.

3 Lowenstein S. Trauma registries: tarnished gold. Ann Emerg Med 1996; 27: 389-391.

4 Kobusingye OC, Ronald LR. Hospital-based trauma registries in Uganda. J Trauma 2000; 48: 498-502.

5 Champion HR, Copes WS, Sacco WJ, et al. The Major Trauma Outcome Study: establishing national norms for trauma care. J Trauma 1990; 30: 1356-1365.

6 The abbreviated injury scale 1990 revision. Des Plaines, III: American Association for the Advancement of Automotive Medicine, 1990.

(Received 23 Feb 2006, accepted 22 Aug 2006)

$\square$ 